\title{
Dispatching active distribution networks through electrochemical storage systems and demand side management
}

\author{
Luca Fabietti, Tomasz T. Gorecki, Emil Namor, Fabrizio Sossan, Mario Paolone, Colin N. Jones
}

\begin{abstract}
In this paper, the problem of dispatching the operation of a distribution feeder comprising a set of heterogeneous resources is investigated. In particular, the main objective is to track a 5-minute resolution trajectory, called the dispatch plan that is computed one day before the beginning of operation. During real-time operation, due to the stochasticity of part of the resources in the feeder portfolio, tracking errors need to be absorbed in order to track the committed dispatch plan. This is achieved by modulating the power consumption of a grid-connected battery energy storage system (BESS) and of the HVAC system of a commercial controllable building (CB). To this end, a hierarchical multi-time-scale controller is designed to coordinate the two entities while requiring a minimal communication infrastructure.

The effectiveness of the proposed control framework is demonstrated by means of a set of full-day experimental results on the $20 \mathrm{kV}$ distribution feeder of the EPFL campus that is comprised of: 1) a set of uncontrollable resources represented by 5 office buildings $(350 \mathrm{kWp})$ and a roof-top $P V$ installation $(90 \mathrm{kWp}) 2$ ) a set of controllable resources, namely, a gridconnected BESS (720kVA-500kWh), and a fully-occupied multizone office building $(45 \mathrm{kWp})$.
\end{abstract}

\section{INTRODUCTION}

The electric grid is undergoing a substantial change towards a more sustainable fossil-free configuration which has motivated a rapid and significant increase of renewable production into the generation mix. However, renewable energy sources are inherently uncertain and volatile which poses new challenges to the classic control paradigm of the power grid.

In order to guarantee the proper and safe functioning of the power grid a set of power reserves are typically kept on standby and activated to compensate for both normal fluctuations and major contingencies. Historically, these reserves were represented mainly by highly-responsive power plants, e.g. hydro units. In a scenario where both consumption and production are more uncertain and difficult to predict, the need for these reserves is expected to increase drastically in the near future [1]. Opposed to this existing paradigm, in recent years, both the academic and the industrial community have shifted their attention to a more decentralized control scheme where the proper function of the entire system is achieved by controlling smaller portions of the electric grid. The main idea is then to attain controllability on a local scale by coordinating heterogeneous resources such as storage elements, flexible loads, and distributed generation. In this direction, many contributions have appeared for both demand response (DR) programs at different time scales [2]-[9] as well as aggregation of heterogeneous resources offering primary, secondary, or tertiary reserves to the power grid.
Another paradigm, first introduced in the recent contribution [10], is that of the so-called dispatchability of distribution feeders where the main target is to achieve virtually perfect dispatchability of a set of devices consisting of uncontrollable loads and distributed generation (prosumers). To this aim, the proposed framework requires computing one day in advance a forecast power profile for the aggregated prosumers. During real-time operation, in order to track the committed profile, a Battery Energy Storage System (BESS) is operated by controlling its power injection in order to absorb any errors in the forecasts. The effectiveness of such a control scheme was experimentally validated using a $720 \mathrm{kVA} / 500 \mathrm{kWh}$ BESS to dispatch a $20 \mathrm{kV}$ distribution feeder characterized by both uncontrollable power consumers and distributed photovoltaic generation. Clearly, the success of such a control scheme relies on two factors: 1) an accurate forecasting tool for predicting the power profile of the prosumers one day in advance; 2) the capacity/power specifications for the BESS. For a given maximum prosumer power, it is desirable to achieve dispatchability with the smallest possible battery, as BESS are expensive devices despite, in the recent years, a decreasing trend has been observed [11].

In this paper, we extend the concept proposed in [10] by considering an additional degree of freedom during real-time operation. In particular, the benefit of having a smart commercial building within the prosumer portfolio is investigated. The main reasons for considering commercial buildings over other types of loads is dictated by the following considerations: 1) Commercial buildings are typically characterized by a large thermal inertia that can be naturally exploited to defer their power consumption without affecting occupant comfort; 2) Most commercial buildings are already equipped with energy management systems that could facilitate communication and allow simpler variation of their energy consumption.

The contribution of the present manuscript is then to propose a hierarchical multi-time-scale Model Predictive Controller (MPC) to achieve dispatchable operation by coordinating the BESS and the CB. The MPC controller is designed with the main objective of keeping the battery at a scheduled level by shifting the building consumption. This is achieved while still enforcing both comfort and operational constraints for the building, as well as for the battery.

The effectiveness of the method is demonstrated by means of a set of full-day experimental runs using the same utilityscale electric battery of [10], a fully-occupied smart office building, conventional uncontrollable loads, and a roof-top 


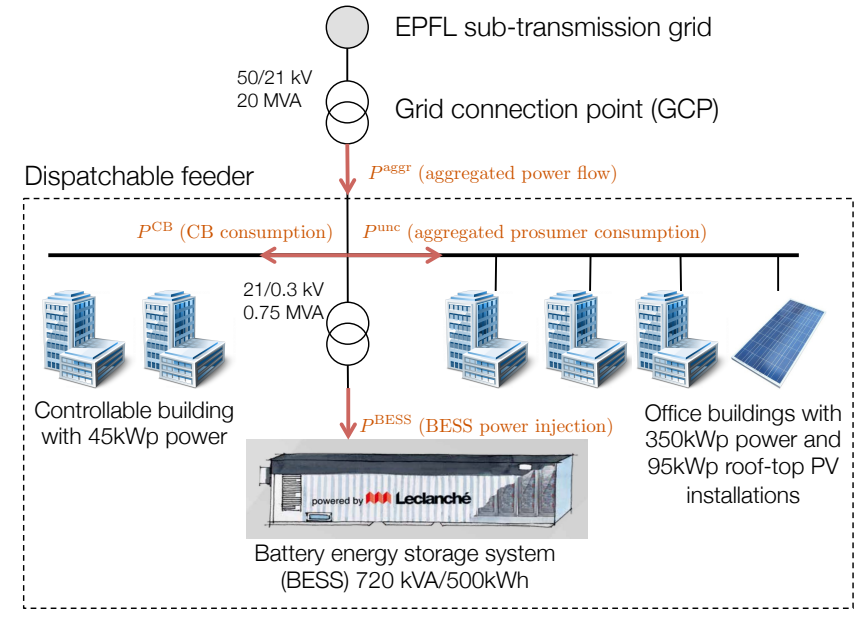

Fig. 1. The experimental setup of Section $\mathrm{V}$ that is used to validate the proposed control architecture. It comprises an MV feeder with a set of heterogeneous uncontrollable buildings, roof-top PV installations, a BESS, and a controllable office building. The control framework requires minimal invasive monitoring infrastructure, namely the aggregated power flow at the GCP, the battery power injection, and the buildings consumption (both controllable and uncontrollable)

PV generator.

The rest of the paper is structured as follows. In Section II we present the formulation of the dispatchability problem. Section III and Section IV describe the day-ahead and realtime operation. Section V describes the experimental results. Finally, Section VI summarizes the contribution of the paper and proposes future improvements and directions.

Notation: Throughout the article, $\mathbb{R}^{n}$ denotes the $\mathrm{n}$ dimensional real space, upper case letters are used for matrices and lower case for vectors. $a_{i}$ represents the value of vector $a$ at time $i$ whereas, bold letters are used to denote sequences over time, e.g., $\mathbf{a}=\left[a_{0}^{T}, a_{1}^{T}, \ldots, a_{N-1}^{T}\right]^{T}$. We use the notation $\hat{\mathbf{P}}^{\text {res }}$ to denote the predicted dispatch profile for the resource, res.

\section{Problem Statement}

The main objective of this paper is to ensure that the aggregated power consumption of a cluster of heterogeneous resources follows a pre-established power profile.

The considered scenario is well exemplified by the considered experimental platform (Figure 1), and it is comprised of:

- a set of heterogeneous, possibly unknown, resources. This would typically comprise both uncontrollable loads, as well as distributed renewable generation, e.g. photovoltaic generators;

- a grid-connected controllable battery energy storage system;

- a relatively large commercial building already equipped with a Building Energy Management System (BEMS).

Referring to Figure 1, in the following we consider the following notation: $\mathrm{P}^{\text {aggr }}$ is the aggregated power consumption at the GCP, $\mathrm{P}^{\mathrm{BESS}}$ is the bidirectional real power flow of the BESS, $\mathrm{P}^{\mathrm{CB}}$ is the power consumption of the controllable building and, $\mathrm{P}^{\mathrm{unc}}$ is the aggregated power consumption of

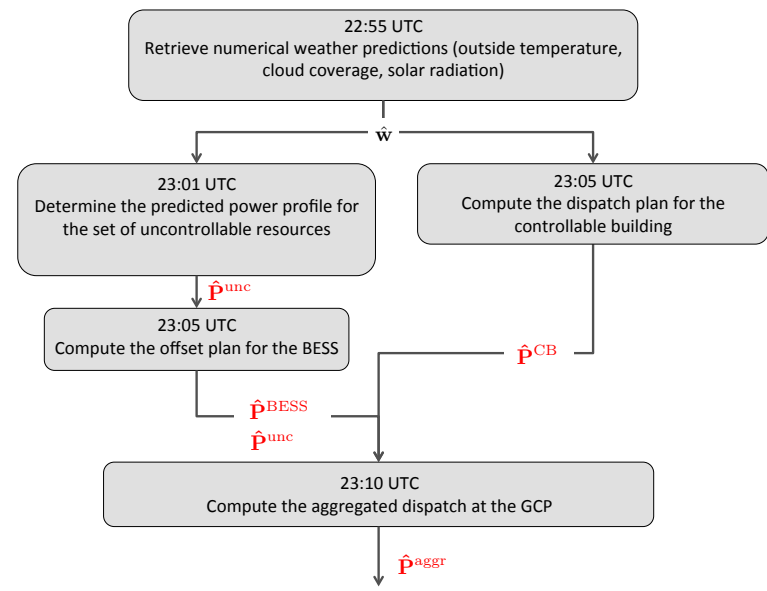

Fig. 2. Flow chart for the day-ahead operation phase.

the uncontrollable resources, also referred as prosumers. For all resources, we assume a passive sign notation, i.e., positive power values correspond to consumption, whereas negative values correspond to power injection towards the GCP. Also, we assume the local distribution feeder to have adequate ample capacity and characteristics so as to operate within its voltage lines and technical constraints.

The dispatchability framework is comprised of two separate phases that are referred to as day-ahead operation, and real-time operation.

- Day-ahead operation: Each day at a pre-specified time ${ }^{1}$, an aggregated power profile for all elements connected at the Grid Connection Point (GCP) is computed for the following day, starting at 00.00 UTC. The power profile, called dispatch plan, is a consumption profile with a 5-minute resolution that the feeder commits to track during real-time operation.

- Real-time operation: Starting at 00.00 UTC, the committed dispatch plan is tracked as closely as possible. To correct for any forecasting error, the dispatch feeder operator can modulate both the power injection of the BESS, as well as the consumption of the flexible loads.

\section{DAY AHEAD PROBLEM}

In this section, the strategy to compute the day-ahead power plan is mathematically formalized. The main objective during day-ahead operation is to compute an accurate dispatch plan (small forecasting error), while, at the same time, making sure to have enough local reserve capabilities to absorb possible forecasting errors. In the absence of global constraints, each independent element of the cluster can compute its own dispatch plan in accordance with its particular objective. The overall power profile is then reconstructed as the sum of the individual contributions as shown in Figure 2.

The predicted dispatch plan at the GCP, for each 5-minute interval, $i$, in the following day, can be written as follows:

\footnotetext{
${ }^{1}$ In this paper, it has been arbitrarily chosen to be one hour before the beginning of real-time operation
} 
Problem 1 (Controllable Building Day-Ahead Problem):

$$
\hat{\mathrm{P}}_{i}^{\mathrm{aggr}}:=\hat{\mathrm{P}}_{i}^{\mathrm{unc}}+\hat{\mathrm{P}}_{i}^{\mathrm{CB}}+\hat{\mathrm{P}}_{i}^{\mathrm{BESS}} \quad i=1, \ldots, 288
$$

where, according to the previously introduced notation, $\hat{\mathrm{P}}_{i}^{\mathrm{unc}}$ represents the predicted power profile for the uncontrollable resources, $\hat{\mathrm{P}}_{i}^{\mathrm{CB}}$ is the dispatch plan of the $\mathrm{CB}$, and $\hat{\mathrm{P}}_{i}^{\mathrm{BESS}}$ is the offset plan to restore the SOC of the BESS to a suitable level.

In the following sections, the procedure to compute each term in (1) is detailed.

\section{A. Uncontrollable Resources Day-ahead Prediction}

We consider a set of heterogeneous resources (uncontrollable loads, distributed generators) with the aim of computing a power profile for the following day so as to minimize the forecast error. The exact procedure to obtain the day-ahead prosumption forecast is the same as the one proposed in [10] and it is not a contribution of this paper. For this reason, in the following the outcomes of the forecasting algorithm are simply stated:

- A set of scenarios, $\Omega$, that is constructed using a completely data-driven approach:

$$
\Omega=\left\{\mathbf{L}_{1}, \ldots, \mathbf{L}_{N_{\text {scen }}}\right\}
$$

where each element $\mathbf{L}_{t}$ represents a power profile realizations, with a 5-minute resolution, that is likely to happen the following day . $N_{\text {scen }}$ determines the number of scenarios in $\Omega$ and it is a user-defined parameter.

- An expected prosumption, $\hat{\mathbf{P}}^{\text {unc }}$, calculated as the component-wise average for all scenarios in $\Omega$.

\section{B. Computation of BESS offset profile}

The offset profile, $\hat{\mathbf{P}}^{\text {BESS }}$, has the objective of restoring a suitable amount of up/down regulation capacity in the BESS. It is computed by a robust optimization problem with the objective of making sure that BESS capacity and power constraints are respected in the extreme cases of the demand realization, which are extracted from the set $\Omega$, given by the forecasting tool. Additional required information are the expected prosumer profile, $\hat{\mathbf{P}}^{\text {unc }}$, and the estimated BESS $\mathrm{SOC}$ at the beginning of the day of operation $\mathrm{SOC}_{0}$. More information can be found in [10].

\section{Controllable Building Day-Ahead Formulation}

In the proposed framework the controllable building commits to offer flexibility during real-time operation. For this reason, the dispatch plan is conceived so as to maximize its reserve capabilities while still enforcing all comfort and operational constraints. The day-ahead problem for the controllable building reads:

$$
\begin{aligned}
& \qquad \hat{\mathbf{P}}^{\mathrm{CB}}=\arg \min \left\{\sum_{i=1}^{N}\left\|y_{i}-T_{\mathrm{ref}}\right\|_{2}\right\} \\
& \text { s.t. } \\
& \quad x_{i+1}=A x_{i}+B_{u} u_{i}+B_{w} \hat{w}_{i} \\
& \quad y_{i}=C x_{i} \\
& \left|y_{i}-T_{\mathrm{ref}}\right| \leq \gamma_{i} \\
& u_{i} \in \mathbb{U}=[0,1]^{n_{u}} \\
& \hat{\mathrm{P}}_{i}^{C B}=h\left(u_{i}\right)
\end{aligned}
$$

for $i=0, \ldots, 288$.

Equations (2) and (3) characterize the dynamical thermal behaviour of the building. In particular, at time $i, x_{i} \in \mathbb{R}^{n_{x}}$ represents the state of the system, $u_{i} \in \mathbb{R}^{n_{x}}$ are the command inputs to the HVAC system (set-points, ON/OFF sequences, etc.), and $w_{i} \in \mathbb{R}^{2}$ is the vector of external disturbances affecting the system such as outside temperature and solar radiation. At time of decision, the initial state, $x_{0}=x(0)$ is known either through direct measurements or by standard state estimation techniques. The indoor temperature in all rooms at time $i$ is obtained by the simple map (3).

Equation (4) enforces an adequate level of comfort for occupants. In particular, we impose that the indoor room temperature should not deviate from a predefined temperature reference, $T_{\text {ref }}$ by more than $\gamma_{i}$ which is a possibly time-varying user-defined parameter.

Equation (5) captures the physical limitations for the equipment. Finally, equation (6) determines the relation between control inputs and overall power consumption of the building.

We assume the disturbance vector, $\hat{\mathbf{w}}$, to be perfectly known by means of weather forecasts. Nevertheless, we would like to stress that there is no conceptual hurdle in extending the presented formulation to explicitly take into account the uncertainty in the weather.

Remark 1: The flexible building dispatch plan is computed in a completely independent fashion from the BESS offset plan, i.e., no communication is required during dayahead operation.

For this reason, the building aims at maximizing its flexibility by simply forcing the indoor temperatures to be in the most comfortable situation, i.e., lay in the middle of the comfort constraints. The rational behind this choice is that, as no information are available at time of decision regarding the forecasting error, the building prepares to equally absorb both negative and positive deviations.

\section{REAL TIME OPERATION}

This section details the real-time controller which represents the main novelty of the present work.

At the beginning of new day operation, i.e. 00.00 UTC, the task of the feeder operator is to precisely track, on a 5-minute resolution, the pre-computed dispatch plan, $\hat{\mathbf{P}}^{\text {aggr }}$. 


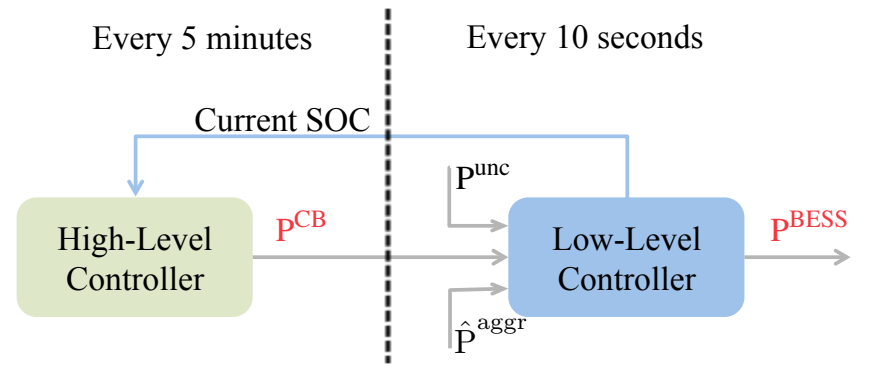

Fig. 3. Time separation for the overall real-time controller. Every slow time interval, the current SOC is transmitted by the BESS operator to the High-Level controller which determines its next action in order to maneuver the SOC. On a faster time resolution (10 seconds), the Low-Level controller measures the power realizations of both the controllable element, $P^{\mathrm{CB}}$, and the uncontrollable prosumer, $P^{\text {unc }}$ and computes the BESS power injection for the following fast time interval, to track the committed dispatch plan, $\hat{P}^{\text {aggr }}$. Legend: Grey lines correspond to measured quantities, blue lines to transmitted information and, red terms represent decision variables at each controller level.

To achieve this aim, it is intuitive to understand that the real-time controller should modulate the power consumption of the two resources at a faster pace $(10 \mathrm{sec}$.). However, due to the physical limitations of the equipment, for most commercial buildings, it is not possible to control the power consumption of the HVAC system with such a granularity. For this reason, in this section, we present the design of a hierarchical multi-time-scale controller, sketched in Figure 3 , that aims at exploiting the synergy between the two controllable elements. On the one side, the BESS represents the master element which ultimately delivers the dispatchable service by correcting forecasting errors at a sub-minute time-scale; on the other side, the smart building operates at a slower time scale and its main goal is to try to restore the SOC of the BESS while respecting its operational constraints. The overall tracking problem is then solved at two well-separated timescales: a Low-Level MPC controller operating at a fast sampling time (10 sec.) continuously computes the power injection of the BESS so as to track the committed dispatch plan; at a slower resolution (5 min.), a High-Level MPC problem is solved to determine the power modulation for the smart building in such a way to restore the SOC of the battery. The advantage of such a configuration is that the only required information to be exchanged during real-time operation is represented by the current SOC of the battery at each slow time sampling.

\section{A. High-Level Controller}

In this section, we describe the control problem solved at the building node with a 5-minute time sampling over a prediction horizon, $N$. The objectives of this controller are: 1) guarantee a high level of comfort for occupants of the building 2) modulate its power consumption with respect to the individual committed power profile, $\hat{\mathbf{P}}^{\mathrm{CB}}$, in order to maneuver the SOC of the BESS to a reference value.

To accomplish the aforementioned tasks, a receding horizon predictive control (MPC) problem is solved at each iteration. The main steps for the MPC algorithm are the following:

1) Retrieve the most recent weather forecast over the considered prediction horizon, $N$. This includes outside temperature, global horizontal irradiance, and cloud coverage.

2) Obtain from weather forecast the solar irradiance on each surface of the building. This step is performed considering both the geometric properties of the building, as well its location and the forecast cloud coverage.

3) Combine the previous two steps to form the vector of disturbances, $\hat{\mathbf{w}}$, over the prediction horizon.

4) Reconstruct the current state of the system, $x(0)$ by means of a Kalman filter.

5) Retrieve the current state of the battery, $\operatorname{SOC}(0)$.

6) Solve the following MPC problem:

Problem 2 (Controllable building real-time operation):

$$
\begin{aligned}
& \mathbf{P}^{\mathrm{CB}}=\arg \min \left\{\sum_{i=1}^{N}\left\|y_{i}-T_{\mathrm{ref}}\right\|_{2}^{2}\right. \\
& \left.+\rho \underset{\epsilon_{i}^{\text {unc }}}{\mathbb{E}} \sum_{i=1}^{N}\left\|\mathrm{SOC}_{i}-\mathrm{SOC}_{i}^{\mathrm{ref}}\right\|_{2}^{2}\right\}
\end{aligned}
$$

s.t.

$$
\begin{aligned}
& x_{i+1}=A x_{i}+B_{u} u_{i}+B_{w} \hat{w}_{i} \\
& y_{i}=C x_{i} \\
& \left|y_{i}-T_{\text {ref }}\right| \leq \gamma_{i} \\
& u_{i} \in \mathbb{U}=[0,1]^{n_{u}} \\
& \mathrm{P}_{i}^{\mathrm{CB}}=h\left(u_{i}\right) \\
& \mathrm{SOC}_{i+1}=\alpha \mathrm{SOC}_{i}+\mathrm{P}_{i}^{\mathrm{BESS}} \\
& \mathrm{P}_{\min }^{\mathrm{BESS}} \leq \mathrm{P}_{i}^{\mathrm{BESS}} \leq \mathrm{P}_{\max }^{\mathrm{BESS}} \\
& \mathrm{P}_{i}^{\mathrm{BESS}}=\left(\hat{\mathrm{P}}_{i}^{\mathrm{CB}}-\mathrm{P}_{i}^{\mathrm{CB}}\right)+\hat{\mathrm{P}}_{i}^{\mathrm{BESS}}+\epsilon_{i}^{\mathrm{unc}} \\
& \epsilon_{i}^{\mathrm{unc}} \sim \mathcal{D}_{\epsilon_{i}}
\end{aligned}
$$

for $i=1, \ldots, N-1, x_{0}=x(0), \operatorname{SOC}_{0}=\operatorname{SOC}(0)$.

Equations (7) to (11) determine, as in the dispatch problem 1 , the feasible input trajectories that the building can support over the prediction horizon without violating its comfort and operational constraints. Equation (12) describes the dynamics of the BESS element ${ }^{2}$. In (13) we enforce the battery injection power to respect its maximum and minimum limitations.

In equation (14), the constraint which underpins the whole proposal is formulated. It is obtained by simply re-arranging the tracking constraint $\hat{P}_{i}^{\mathrm{aggr}} \equiv P_{i}^{\mathrm{aggr}}$ as a function of $P_{i}^{\mathrm{BESS}}$. Essentially, this equality constraint states that the power injection of the battery can be controlled by acting on the difference between the committed dispatch plan and the actual power consumption of the CB. In a nutshell, depending on the value of the relative weight, $\rho$, the building aims at minimizing the deviation of the SOC from a predefined reference while attempting to drive the room temperatures to the most comfortable configuration. This is accomplished

\footnotetext{
${ }^{2}$ The loss coefficient, $\alpha$, has been estimated using historical data.
} 


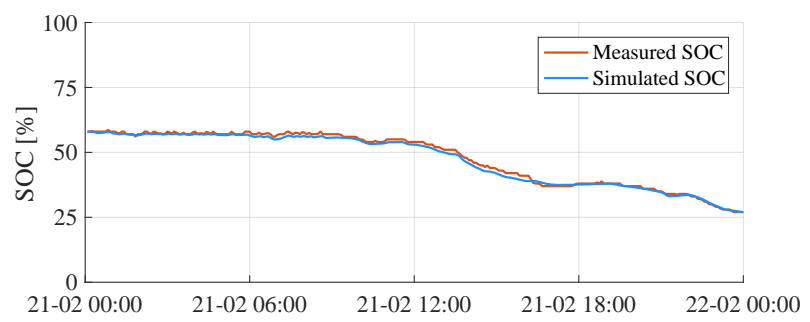

Fig. 4. Validation of the battery model used in Problem 2. For the considered validation data set, the model fit is $\sim 93 \%$.

by modifying its power consumption with respect to its dispatch plan to lead to a net charging/discharging event. Please note that in order to account for the uncontrollability of the prosumers, an uncertain term $\epsilon_{i}^{\text {unc }}$ is also added. The probability distribution, $\mathcal{D}_{\epsilon_{i}}$, of this term can be estimated from historical data using standard available techniques. In the present work, for the sake of simplicity, we consider a vanishing persistent estimator, i.e., the term $\epsilon_{i}^{\mathrm{unc}}$, at time $i$, is assumed to be expressed as

$$
\hat{\epsilon}_{i}^{\mathrm{unc}}=\xi^{i} \bar{\epsilon}
$$

where $\bar{\epsilon}$ represents the last measured forecast error and $\xi(0.8$ in the experiments ${ }^{3}$ ) is a forgetting factor which determines the rate at which the error is assumed to vanish.

Remark 2: In the High-Level controller formulation, a simple linear reservoir model for the BESS was considered (12). Clearly the model does not capture complex dynamics of the battery such as charging/discharging losses, thermal effects, etc. that can be incorporated using a non-linear first-principle-based model [12]. Nevertheless, due to the hierarchical structure of the controller, the higher layer does not necessitate an exhaustive description of the BESS internal states which are instead considered at the lower layer. On the contrary, the simple linear model is exploited by the MPC to obtain a coarse prediction of the future SOC as a function of the power of the injected power according to (12) and (14). Moreover, as it can be noticed in Figure 4, the considered model can reasonably capture the main trends of the SOC, achieving a fit of roughly $93 \%$ on the validation data. Tra

With the considered assumptions, Problem 2 is a deterministic quadratic programming problem that can be easily and efficiently solved in real-time using non-commercial solvers.

\section{B. Low-Level Controller}

After the building re-dispatch action performed by the High-Level Controller (which acts at 5-minute resolution), it is still necessary to compensate for the mismatch between the real-time realization and the aggregated dispatch plan. This is accomplished by the Low-Level controller, which determines the active power set-point of the BESS four quadrant power converter. Unlike the power consumption of building space heating systems which cannot be modified at a fast pace, BESS's injections can be typically controlled at

\footnotetext{
${ }^{3}$ The value for $\xi$ was chosen based on historical realizations of the forecast error.
}

a high-frequency and are, therefore, suitable to perform fine power/energy adjustments. The Low-Level controller is the MPC algorithm described in [10] and is not a contribution of this work. In summary, it consists in solving an optimization problem at $10 \mathrm{~s}$ resolution on a 5 minute shrinking horizon, from the current time period until the end of the dispatch interval. The cost function is given by minimizing the energy mismatch between real-time realization (progressively known from real-time measurements) respect to the dispatch plan while being subject to BESS operation constraints on DC voltage, DC current and SOC limits. At this stage, the BESS is operated at unitary power factor, in other words there is no reactive power injection.

\section{EXPERIMENTAL IMPLEMENTATION}

In this section we present a series of experiments conducted on a real-scale MV distribution grid in the EPFL campus. Experiments have been performed in the period from December 2016 to March 2017. First, we provide more details regarding each involved element.

1) Battery electric energy system (BESS): We consider a Leclanché grid-connected Lithium Titanate BESS characterized by a $720 \mathrm{kVA} / 500 \mathrm{kWh}$ power/capacity ratio. The battery consists of 9 parallel racks (each composed of a 15 modules in series, where each module is composed of a $20 \mathrm{~s} 3 \mathrm{p}$ cell pack), a four quadrant fully-controllable DC/AC converter, and a $0.3 / 20 \mathrm{kV}$ step-up transformer. The whole system is placed in a temperature controlled environment.

2) Non-controllable units: The second key element in the considered scenario, which also represents the main source of uncertainty, is composed of: 1) an aggregation of noncontrollable buildings with a $350 \mathrm{~kW}$ peak consumption; 2) a $95 \mathrm{kWp}$ root-top PV installation. The composite power for both the uncontrollable units and the BESS is measured at the feeder by means of high-frequency high-precision phasor measurements units (PMU) [13].

3) Controllable Building: The last element is represented by a controllable subsection of a fully-occupied laboratory in the EPFL campus. The experiments were conducted in four office rooms which account for a total area of 115 sq. meters ( 1200 sq. ft.). Rooms are labeled according to their exposure to the sun. Rooms NW, N, SW are individual offices whereas room SE is an open-space office occupied by six people. Each room is equipped with a commercial electric heater. The heaters are rated at $P_{\max }=1950$ Watts, summing up to a total installed capacity of 7800 Watts. All heaters are centrally controlled by the real-time operation controller of Problem 2, running in MATLAB, which collects room temperatures from the web database, weather forecast, and the last state estimate and decides both the aggregated power consumption for the next 5 minutes, as well as the allocation across the 4 rooms.

The model of the building used in both Problem (1) and (2) is obtained through completely data-driven identification techniques. More details in [5].

a) Scaling: As already mentioned, the proposed framework is tailored to the coordination of a medium/large-size 


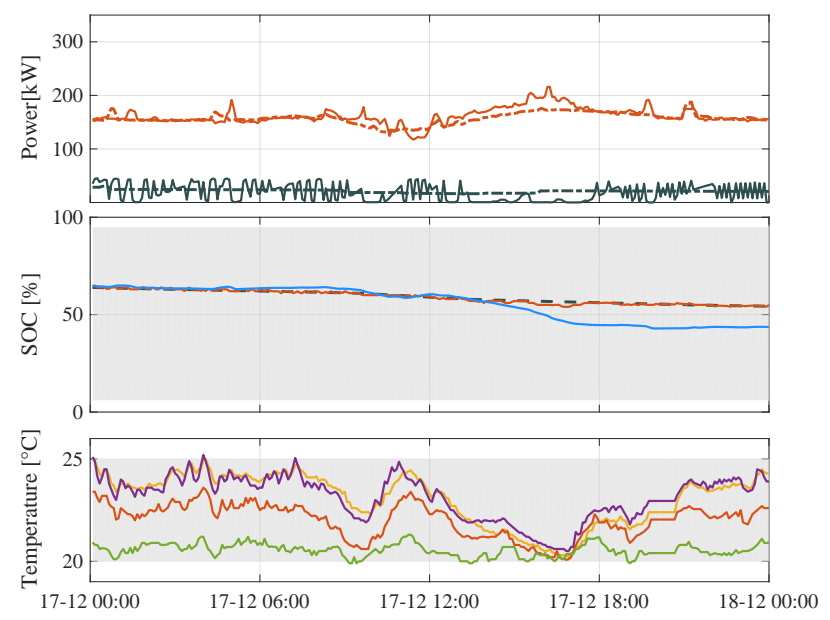

(a) Day 1: Dispatch tracking in presence of mild forecasting errors

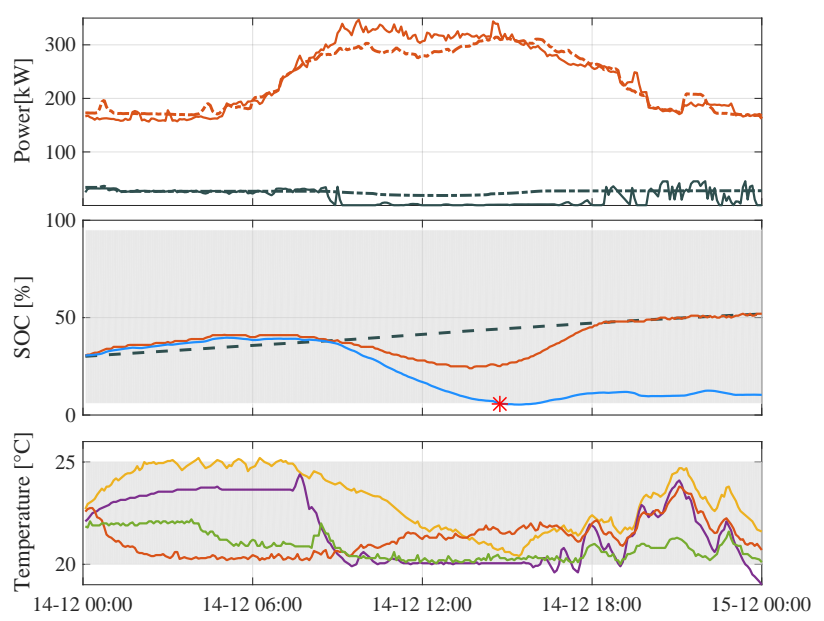

(b) Day 2: Dispatch tracking in presence of extreme forecasting errors

Fig. 5. Real-time operation for the dispatch tracking. Upper: The black dashed line represents the dispatch plan for the $\mathrm{CB}, \hat{\mathbf{P}}^{\mathrm{CB}}$, whereas its actual realization, $\mathbf{P}^{\mathrm{CB}}$, is shown using a black solid line. Similarly, for the uncontrollable resources, the dashed orange line represents the day-ahead predicted power profile, $\hat{\mathbf{P}}^{\text {unc }}$ and the solid line its measured value, $\mathbf{P}^{\text {unc }}$. Middle: The black dashed line represents the SOC reference, SOC ${ }^{\text {ref }}$. The experimental realization of the SOC is displayed in orange. The blue line is the simulated SOC in absence of the controllable building as previously explained. Lower: Temperature variation for the difference zones of the controllable building. Each color corresponds to the measured temperature in a zone. In both the middle and lower plots, the grey area represents the allowed ranges for the plotted quantities

CB and a utility-scale BESS. However, the considered flexible demand accounts for a $\sim 8 k W$ peak consumption which represents only $2 \%$ of the $350 \mathrm{~kW}$ peak of uncontrollable units. To have a rated power comparable with the one of the uncontrollable resources and, therefore, being able to draw meaningful conclusions on the impact of a controllable load in the dispatchable feeder framework, we decided to virtually scale up by a factor of 6 the power consumption of the building, leading to a maximum peak of $45 \mathrm{~kW}$. In addition, the scaling factor was chosen in order to have the minimum size to prevent previously experienced failures. In particular, among the set of experiments with no controllable building [10], we selected the days were the dispatchable feeder operator failed to track the committed dispatch plan due to a complete charge/discharge of the BESS. A simulationbased analysis was then performed in which the size of the controllable building was slowly increased until the aggregated system manages to successfully track the dispatch plan. Finally, we highlight that scaling up the rated power of the $\mathrm{CB}$ is simply equivalent to consider a larger building (or more rooms) characterized by the same thermal and electrical dynamics.

\section{A. Results and discussion}

In this section, we present a set of experimental results obtained in the period from December 2016 to March 2017. To show the effectiveness of the novel scheme with respect to its previous configuration [10], we consider, as a simple metric, the used SOC needed to achieve dispatchability. Referring to Figure 5, for each day of operation, the following two quantities are compared:

- The actual SOC as it is measured during the experiments (blue solid line),

- The simulated SOC for the battery in absence of the CB (orange solid line). More precisely, as in [10], we reperform in simulation the experiment in the case where the only degree of freedom during real-time operation is represented by the BESS. Hence, starting from its actual initial condition, the SOC is propagated through its dynamical model with the input being the dispatch error for the uncontrollable elements.

In the following, we select two particular days of operation which represent the spectrum of results obtained during the experimental campaign. We classify them depending on the observed forecasting error: Day 1 represents a situation where the forecasting error is moderate and, therefore, the BESS alone would have accomplished to track the dispatch plan. On the contrary, Day 2 is characterized by a significant forecasting error. In this case, the presence of the second degree of freedom is necessary to achieve dispatchability.

Figure 5(a) depicts the real-time operation for Day 1. Thanks to a quite accurate prediction plan for the heterogeneous resources, the BESS alone can easily compensate for the prediction error. In fact, in this case, the simulated SOC is well within its operational limits. Nevertheless, the battery requirements are drastically reduced when the controllable building is considered. This is particularly evident considering the central hours of the day, between 12:00 and 17:00. In fact, due to excessive prosumer consumption, the BESS would have experienced a partial discharge. On the contrary, the proposed algorithm prevents the discharge by exploiting the added flexibility represented by the thermal inertia of the building. Specifically, as shown in the top plot, the power consumption of the building is lowered for a limited period of time to counterbalance the negative forecasting error. This is done while still preserving a high level of comfort as virtually no temperature violations are observed (lower plot).

The second day of operation, displayed in Figure 5(b), 
TABLE I

STATISTICS OVER ALL 12 EXPERIMENTS CONDUCTED IN THE PERIOD BETWEEN DECEMBER 2016 AND FEBRUARY 2017.

\begin{tabular}{|l|c|c|}
\hline Quantity & BESS & BESS + CB \\
\hline Max. SOC used [kWh] & 262.3 & 149.7 \\
Avg. SOC used [kWh] & 153.4 & 74.5 \\
Max. BESS Power [kW] & 82.51 & 65.23 \\
Avg. Comfort Violation ${ }^{\circ} \mathrm{C}$ & n.a. & 0.08 \\
Max. Comfort Violation ${ }^{\circ} \mathrm{C}$ & n.a. & 0.97 \\
\hline
\end{tabular}

represents a situation in which the presence of the deferrable load is crucial to achieve dispatchability. Once again, due to a significantly higher prosumer consumption between roughly 10:00 and 14:00, the BESS would have incurred a rapid discharge until actually violating its lower constraint which corresponds to $5 \%$ of the total available storage. This particular failing event is displayed in the central plot of Figure 5(b) by a red cross around 15:00. Conversely, as in the previous case, the thermal inertia of the building is successfully exploited to decelerate the rate of discharge of the BESS and, therefore, prevent the failure. As it can be observed in the lowermost plot, in order to absorb the negative forecasting error, the controller almost entirely utilizes the flexibility of the building so that few modest comfort violations are experienced in this case.

As a summary, in both situations Figure 5(a) and Figure 5(b), the presence of the controllable building has two positive effects: on the one hand, it helps to reduce the required capacity for the BESS, and on the other hand it helps to track a predefined SOC reference. These results are achieved while still providing a high level of comfort for the occupants as hard comfort constraints are considered in the MPC formulation 2. Finally, we report in Table I the statistics of all conducted experiments.

\section{CONCLUSION}

This paper considered the problem of ensuring that the aggregated power consumption of a cluster composed of a large $\mathrm{CB}$, a grid-connected battery energy storage system, and a set of non-controllable heterogeneous resources follows a forecast profile, called a dispatch plan, which is established the day before operation. To attain this aim, a hierarchical multi-time-scale control framework to coordinate the BESS and the $\mathrm{CB}$ has been proposed: at the Low-Level the BESS power flow is controlled at a 10 -second resolution so that the power flow at the grid connection follows the committed dispatch plan; the High-Level controller determines the CB power consumption at a 5 minutes time-scale to maintain the BESS SOC at a scheduled level. The effectiveness of the overall control scheme is demonstrated by means of a series of real-life real-scale experiments MV system on the EPFL campus.

\section{REFERENCES}

[1] E. Ela, M. Milligan, and B. Kirby, "Operating reserves and variable generation," Tech. Rep., 2011.

[2] S. Teleke, M. E. Baran, A. Q. Huang, S. Bhattacharya, and L. Anderson, "Control strategies for battery energy storage for wind farm dispatching," IEEE Transactions on Energy Conversion, vol. 24, no. 3, pp. 725-732, Sept 2009 .

[3] M. A. Abdullah, K. M. Muttaqi, D. Sutanto, and A. P. Agalgaonkar, "An effective power dispatch control strategy to improve generation schedulability and supply reliability of a wind farm using a battery energy storage system," IEEE Transactions on Sustainable Energy, vol. 6, no. 3, pp. 1093-1102, July 2015 .

[4] M. Maasoumy, "Flexibility of Commercial Building HVAC Fan as Ancillary Service for Smart Grid," in IEEE Green Energy and Systems Conference (IGESC 2013), Nov. 2013.

[5] L. Fabietti, T. Gorecki, F. Qureshi, A. Bitlislioglu, I. Lymperopoulos, and C. Jones, "Experimental implementation of frequency regulation services using commercial buildings," IEEE Transactions on Smart Grid, vol. PP, no. 99, pp. 1-1, 2016.

[6] T. T. Gorecki, L. Fabietti, F. A. Qureshi, and C. N. Jones, "Experimental demonstration of buildings providing frequency regulation services in the swiss market," Energy and Buildings, vol. 144, pp. 229 - 240, 2017. [Online]. Available: http://www.sciencedirect.com/science/article/pii/S0378778816311616

[7] L. Zhao and W. Zhang, "A geometric approach to virtual battery modeling of thermostatically controlled loads," in 2016 American Control Conference (ACC), Jul. 2016, pp. 1452-1457.

[8] H. Hao, T. Middelkoop, P. Barooah, and S. Meyn, "How demand response from commercial buildings will provide the regulation needs of the grid," 2012 50th Annual Allerton Conference on Communication, Control, and Computing, Allerton 2012, pp. 1908-1913, 2012.

[9] Y. Lin, P. Barooah, S. Meyn, and T. Middelkoop, "Experimental Evaluation of Frequency Regulation from Commercial Building HVAC system," IEEE Transaction on Smart Grid, vol. 6, no. no. 2, pp. 776783, March 2015

[10] F. Sossan, E. Namor, R. Cherkaoui, and M. Paolone, "Achieving the dispatchability of distribution feeders through prosumers data driven forecasting and model predictive control of electrochemical storage," IEEE Transactions on Sustainable Energy, vol. 7, no. 4, pp. 1762 1777, Oct 2016.

[11] B. Nykvist and M. Nilsson, "Rapidly falling costs of battery packs for electric vehicles," Nature Climate Change, vol. 5, pp. 329-332, 2015.

[12] E. Namor, D. Torregrossa, R. Cherkaoui, and M. Paolone, "Parameter identification of a lithium-ion cell single-particle model through non-invasive testing," Journal of Energy Storage, vol. 12, pp. 138 - 148, 2017. [Online]. Available: http://www.sciencedirect.com/science/article/pii/S2352152X1630278X

[13] M. Pignati, M. Popovic, S. Barreto, R. Cherkaoui, G. D. Flores, J.-Y. Le Boudec, M. Mohiuddin, M. Paolone, P. Romano, S. Sarri et al., "Real-time state estimation of the epfl-campus medium-voltage grid by using pmus," in Innovative Smart Grid Technologies Conference (ISGT), 2015 IEEE Power \& Energy Society. IEEE, 2015, pp. 1-5. 